\title{
Interaction between As and InP(110) studied by photoemission
}

\author{
H. Oscarsson, Z. Q. He,* L. Ilver, J. Kanski, S. Mankefors, and P. O. Nilsson \\ Department of Physics, Chalmers University of Technology, SE-412 96 Göteborg, Sweden \\ U. O. Karlsson \\ Department of Physics, Materials Physics, Royal Institute of Technology, SE-100 44 Stockholm, Sweden
}

(Received 3 December 1998; revised manuscript received 21 July 1999)

\begin{abstract}
Arsenic deposition on $\operatorname{InP}(110)$ was studied by means of core-level and valence-band photoemission. By systematic spectral decompositions, it was found that the adsorption at room temperature is nonreactive. At elevated temperatures the As $3 d$ spectra show drastic transformations, indicative of a P-As exchange reaction. The data can at this point be interpreted in terms of a recently proposed structure involving an As-covered InAs layer. Upon As redeposition the core-level spectra are clearly different from those observed prior to annealing, which means that the exchange reaction is irreversible. Angle-resolved valence-band spectra from the reacted surface are similar to those from clean $\operatorname{InP}(110)$, but important differences are found, facilitating the identification of surface states.
\end{abstract}

\section{INTRODUCTION}

The adsorption of group-V overlayers on the (110) surface of III-V semiconductors has long been a topic of intensive research. ${ }^{1}$ The ordered V/III-V overlayer structure is considered as a prototype system for investigations of heterointerfaces. Much of the earlier work was concerned with $\mathrm{Sb}$ overlayers, mainly because the exceptionally abrupt and well-defined Sb overlayers on III-V surfaces ${ }^{2}$ provide ideal conditions for investigations of Schottky barrier formation. The recent activities are also concerned with other adsorbates, e.g., As. Besides the motivation of better understanding the interface formation, the As/III-V work is oriented to the study of the chemical reactions between As and the substrates. This has implications for molecular-beam-epitaxy (MBE) growth and surface passivation of III-V semiconductors. $^{3}$

One central point of interest in these studies is the atomic structure of the overlayers. Among the models discussed, the following configurations are the most thoroughly investigated: ${ }^{4}$ epitaxial continued layer structure (ECLS), epitaxial on-top structure, and epitaxial overlapping chain structure. Extensive studies have established the ECLS configuration in the case of $\mathrm{Sb} / \mathrm{GaAs}(110) .{ }^{1}$ In a photoemission study of the As/GaAs(110) system we found, however, features not consistent with this model. ${ }^{5} \mathrm{As} / \mathrm{InP}(110)$ appears to be another case with a deviating structure. In an optical spectroscopy study, combined with $a b$ initio total-energy calculations ${ }^{6}$ it was concluded that an exchange reaction occurs at room temperature between the As and substrate P, leaving an InAs layer on the InP substrate. It was also concluded that this well-ordered phase transforms reversibly into another phase containing a second As overlayer in an ECLS configuration on top of this InAs layer. However, in view of the great electronic similarity between different III-V compounds, and the fact that an exchange process on a (110) surface involves the breaking of three covalent bonds, such reactivity at room temperature appears rather surprising, and further detailed studies are of obvious interest.
In this perspective we have reinvestigated the As/ InP(110) system, both experimentally (by means of photoemission) and theoretically. The theoretical results, involving supercell slab total energy calculations based on the densityfunctional theory within the gradient corrected local-density approximation have been presented in a separate paper. ${ }^{7}$ In short, different unreacted and exchange-reacted model structures were considered. It was found that although the P-Asexchanged configuration is higher in energy than the As/ InP(110) ECLS, the difference between the two systems is indeed small. Thus an exchange reaction at room temperature cannot be excluded on the basis of these calculations.

In the present paper we shall discuss the photoemission data, with an emphasis on the development of the As corelevel spectra after initial deposition, annealing, and redeposition. Core-level photoemission is, in principle, a powerful method for testing this kind of problem. Unfortunately, the hitherto reported photoemission studies ${ }^{8,9}$ were not particularly focused on the P-As exchange aspect. In addition, the valence-band spectra will be presented for the annealed, reacted surface. As a natural consequence of the chemical similarity between the exchanging species, and due to a full adaptation of the exchanged layer to the substrate, the reaction-induced changes in the valence-band spectra are expected to be small. Indeed, the valence-band spectra have been reported to be practically unaffected by the P-As exchange. 9

\section{EXPERIMENT}

The experiments were carried out at beamline 41 at the Swedish national synchrotron radiation facility MAX-lab, where an angle resolving photoelectron spectrometer equipped with low-energy electron diffraction (LEED) is connected to a toroidal grating monochromator. ${ }^{10}$ The As depositions were performed from a conventional $\mathrm{As}_{4}$ Knudsen-type source in a dedicated MBE chamber, directly attached to the photoelectron spectrometer. The sample crystals were in the shape of oriented $n$-type InP bars with 5 
$\times 5-\mathrm{mm}^{2}$ cross section. They were mounted on Mo disks, transferable under ultrahigh vacuum between the MBE and analytical chambers. Clean $\operatorname{InP}(110)$ surfaces were obtained by cleaving, using a commercial knife-anvil setup, located in the transfer chamber interconnecting the analytical and MBE units. Due to the slow thermal characteristics of the $\mathrm{As}_{4}$ source, and limitations imposed by restrictions in the sample transfer procedure, the exposure could not be regulated with accuracy. In particular, despite repeated efforts we were unable to achieve initial As coverages in the submonolayer range. During As evaporation the samples were kept at room temperature. Annealing was performed in the spectrometer chamber by means of a dual filament heater mounted on the backside of the sample holder, and the temperature was estimated via a thermocouple spot welded on the Mo-disk holder. As a result of nonideal thermal contact between sample and thermocouple, the temperature determinations were not very accurate. In the present experiments the desorption temperature of thick As overlayers $\left(350^{\circ} \mathrm{C}\right)$ was used as an internal reference.

The spectra were collected using an angle-resolving, goniometer mounted, hemispherical electrostatic energy analyser with an angular acceptance of $3^{\circ}$. The overall energy resolution was approximately $0.15 \mathrm{eV}$. All data were obtained in $p$-polarization geometry.

\section{RESULTS}

The clean $\operatorname{InP}(110)$ surfaces were optically flat and showed sharp, low-background $1 \times 1$ LEED patterns. Valence-band photoemission spectra were in good agreement with our previous data, ${ }^{11}$ and the In $4 d$ and P $2 p$ corelevel spectra showed the established surface core-level shifts. ${ }^{12}$ After exposure to $\mathrm{As}_{4}\left(p_{\text {beam }} \approx 1 \times 10^{-6} \mathrm{~Pa}\right)$ for periods of $1-5 \mathrm{~min}$, the $1 \times 1$ LEED spots were still discernible, though weak and fuzzy, and on a significantly higher background. From this we conclude that the As overlayer was a few monolayers thick and disordered. In the following we shall focus on the spectral development of the As $3 d$ emission as the sample was subject to repeated periods of thermal annealing. It must be pointed out that we have also recorded the substrate related core-level spectra, but these data will not be discussed here, mainly because the analysis of the $\mathrm{P} 2 p$ emission was found to be inconclusive due to experimental problems (occasionally poor energy resolution at photon energies above $150 \mathrm{eV}$ caused by imaging imperfections of the monochromator grating). The In $4 d$ spectra were qualitatively similar throughout, and were used for monitoring changes in the band bending.

Figure 1 shows the development of the As $3 d$ spectra at different stages of treatment following the initial exposure. The spectra in Figs. 1(b)-1(h) have been subject to small shifts, to account for band bending variations accompanying the surface modifications. The unshifted spectrum in Fig. 1(a) shows the situation in the case of excessive As deposition (quenching all substrate related signals). Along with the data points we show the fitted curves (see below), the deduced spectral components, and the residues. Scale factors are given to indicate how the absolute intensities compare between the spectra [e.g., the absolute intensities of Figs. $1(\mathrm{e}), 1(\mathrm{f})$, and $1(\mathrm{~g})$ are approximately $30 \%$ of that of Figs. 1(b) and 1(h), and about $50 \%$ of Fig. 1(c)]. After annealing to a saturation level (above which no further spectral changes occur), represented by Fig. 1(g), a distinct, very low background $1 \times 1$ LEED pattern was observed, indicative of a well-ordered surface. To test the relative surface sensitivity of the two final As $3 d$ components, spectra were recorded in normal emission and at $50^{\circ}$ off normal (Fig. 2).

Upon a second As exposure, the LEED spots again became much weaker and the background was increased, just as after the first exposure. The As $3 d$ spectrum after this redeposition is shown in Figs. 1(h) and 3. It is clear that the indicated decomposition into the same three components as in the case of the freshly cleaved As exposed surface [compare Figs. 1(b) and 1(h)] is not adequate. After a new sequence of annealings the Fig. 1(g) spectrum was restored. This spectrum turned out to be stable against annealing at temperatures up to about $500{ }^{\circ} \mathrm{C}$. Above this point the As signal was strongly reduced, and the In spectra revealed a high-energy component indicative of a metallic environment. The surface was, however, still optically mirrorlike, and the LEED pattern was practically unaffected high-quality $1 \times 1$. A more detailed study of this surface will be presented elsewhere. For the present discussion it is relevant to show the spectra obtained after prolonged annealing (at up to about $330{ }^{\circ} \mathrm{C}$ ) of this surface in an $\mathrm{As}_{4}$ atmosphere. By this treatment the metallic In component was eventually fully removed. It could also be noted that the P $2 p$ signal was visible at this stage, but much weaker than prior to As redeposition, showing that the substrate was covered by several monolayers of InAs. Figure 4 displays As $3 d$ spectra from this restored surface after a new As redeposition and after subsequent annealing.

In Fig. 5 we show a comparison between sets of valenceband spectra from the freshly cleaved $\mathrm{InP}(110)$ surface and the As-dosed and annealed surface [corresponding to the spectrum in Fig. 1(g)]. Since our purpose is to compare data from different samples, we have chosen to present spectra along the $\bar{\Gamma} \bar{X}$ azimuth [i.e., along the zigzag chains on the (110) surface]. In this way we avoid ambiguities that may arise due to the asymmetry of the surface unit cell. The data from the two surfaces were recorded under identical experimental conditions. A more detailed comparison between spectra at $20^{\circ}$ emission, probing the $\bar{X}$ point, is shown in Fig. 6.

\section{DISCUSSION}

Due to the complexity of the As core-level spectra, it is appropriate to start the discussion by a short description of our data processing. The fittings were performed using a computer routine, by which the peaks are approximated by convoluted Gaussian and Lorentzian functions (the former primarily accounting for the instrumental broadening, the latter for lifetime broadening). It is well known that this type of fitting is not unique, and this is particularly true in cases where the individual spectral components are not clearly visible in the raw data. In order to treat such situations with some confidence, it is necessary to impose restrictions on the fitting parameters. In the present analysis the energy broadening parameters were of course required to be compatible with the instrumental resolution and lifetime widths, and the 


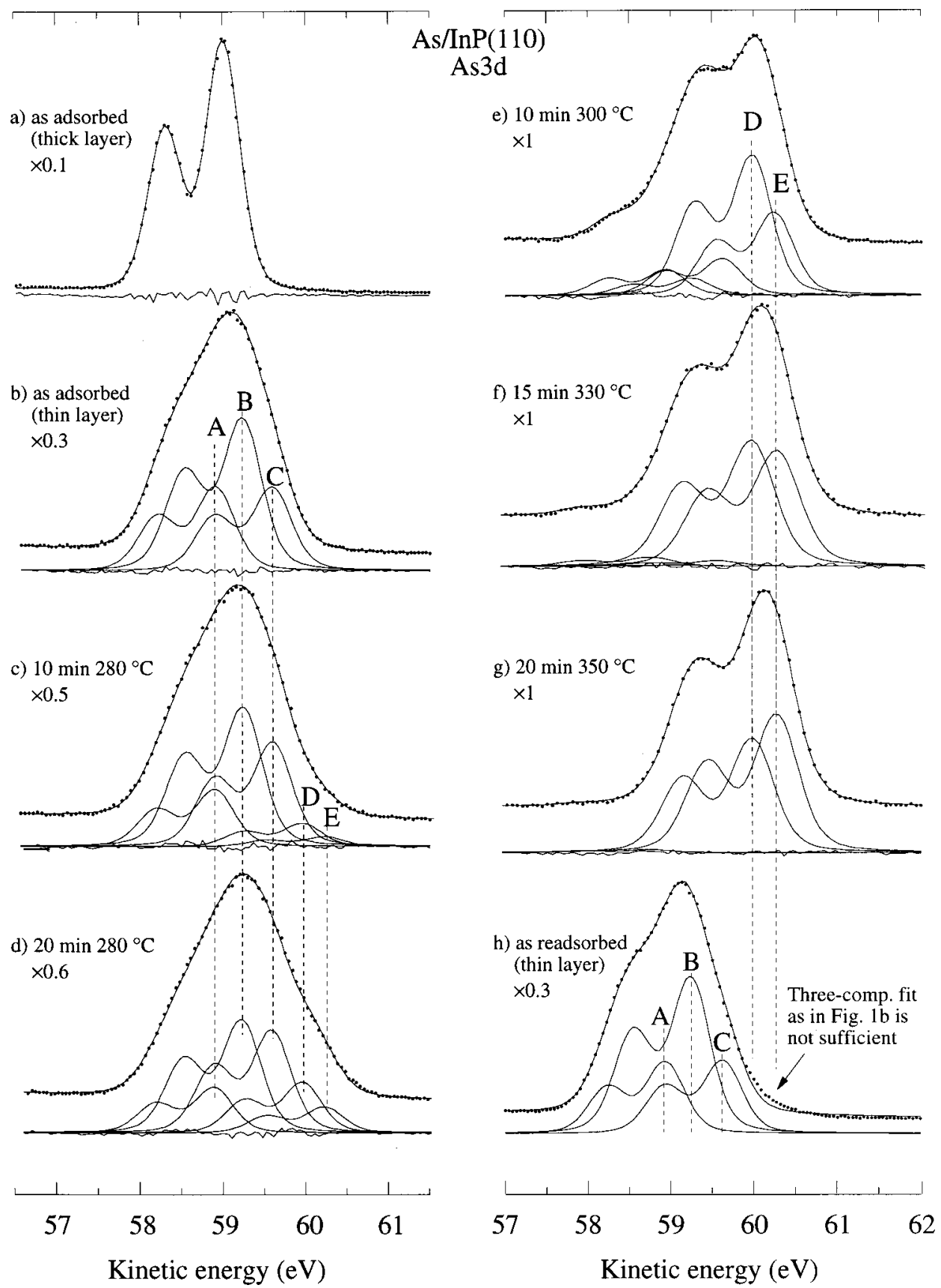

FIG. 1. Normal-emission As $3 d$ core-level spectra from $\mathrm{As}_{4}$-exposed $\mathrm{InP}(110)$ at different stages of annealing. The absolute intensities of (e), (f), and (g) are approximately $10 \%$ of that in (a) $30 \%$ of (b) and (h), $50 \%$ of (c) and $60 \%$ of (d) as indicated by the scale factors in the figure. All core-level spectra in this and the following figures were excited with $105-\mathrm{eV}$ photons. The data points are shown together with fitted curves and spectral decompositions as described in the text. Fitting parameters: $\Delta E_{\mathrm{Gauss}}=0.455 \mathrm{eV}, \Delta E_{\mathrm{Lor}}=0.155 \mathrm{eV}$, and $\Delta E_{\mathrm{s}-\mathrm{o}}=0.695 \mathrm{eV}$. After the final annealing (g) the Gaussian broadening was reduced to $0.42 \mathrm{eV}$, and in the case of a thick As overlayer (a) $\Delta E_{\mathrm{Gauss}}=0.42 \mathrm{eV}$ and $\Delta E_{\mathrm{Lor}}=0.102 \mathrm{eV}$. Note that (h) does not show a fully optimized fit, as discussed in the text.

same parameters were consistently applied to spectra taken at different emission angles. Furthermore, once the presence of the different components was concluded, their relative positions within the spectra were kept fixed. The same set of spectral components was used to fit a large number of spectra, recorded from different samples and under varying experimental conditions. The fitting parameters are given in the figure captions, and in Table I we give the relative energy positions of the different components discussed below.

The spectrum recorded prior to any heat treatment is shown in Fig. 1(b). We note that it is qualitatively similar to the corresponding spectrum of $\mathrm{Sb}$ adsorbed on $\operatorname{GaAs}(110),{ }^{13}$ in which case the overlayer is established to be nonreacted. The spectrum is rather broad and structureless, and it can be well fitted by two spin-orbit-split components. ${ }^{9}$ The fact that such fitting would involve strongly broadened components could be acceptable in view of the disordered overlayer structure. On the other hand, by imposing restrictions regarding the broadening parameters $\left(\Delta E_{L}=0.155 \mathrm{eV}\right.$, and $\Delta E_{G}$ matching the values employed in the analysis of the parallel In $4 d$ spectra), we find that the fitting requires at least three components. Indeed, the spectral modifications observed 

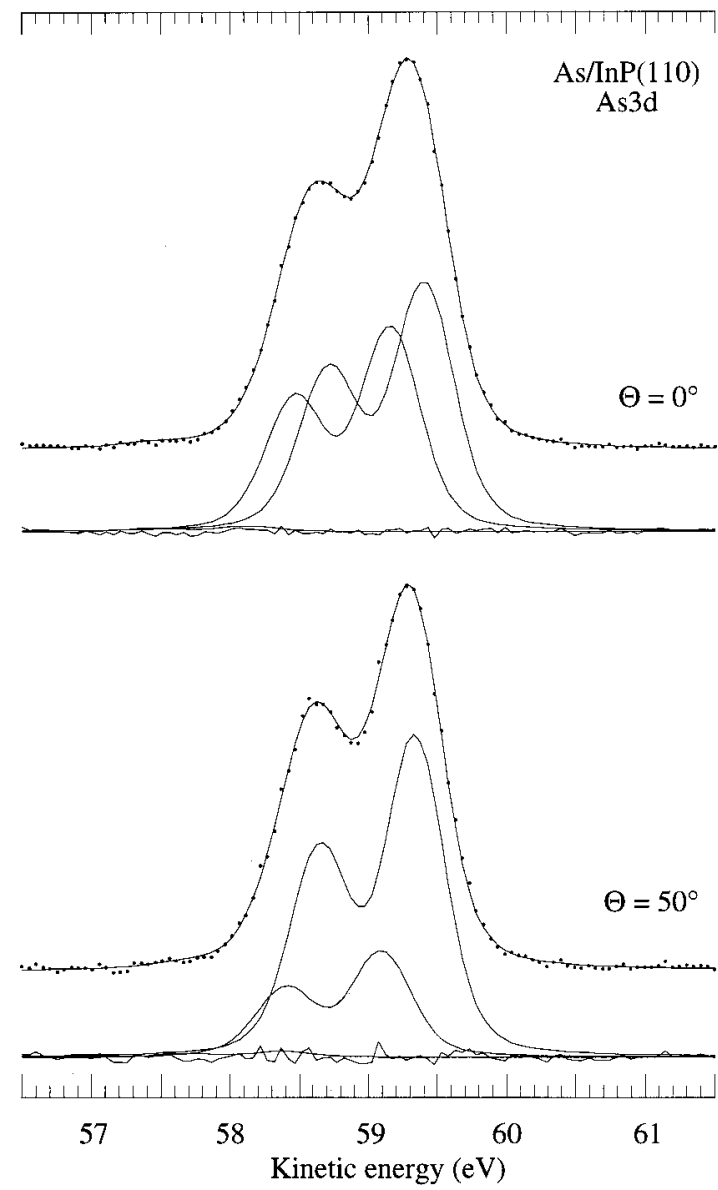

FIG. 2. As $3 d$ spectra for annealed $\operatorname{As} / \operatorname{InP}(110)$ at $0^{\circ}$ [the same as Fig. $1(\mathrm{~g})]$ and $50^{\circ}$ emission angles. Fitting parameters as in Fig. 1.

during the subsequent annealings support this decomposition. We also find that spectra of thick As overlayers (quenching all substrate-related signals) are well fitted by a single, narrow $\left(\Delta E_{L}=0.102 \mathrm{eV}, \Delta E_{G}=0.42 \mathrm{eV}\right)$ spin-orbitsplit component [Fig. 1(a)], indicating that As atoms at some

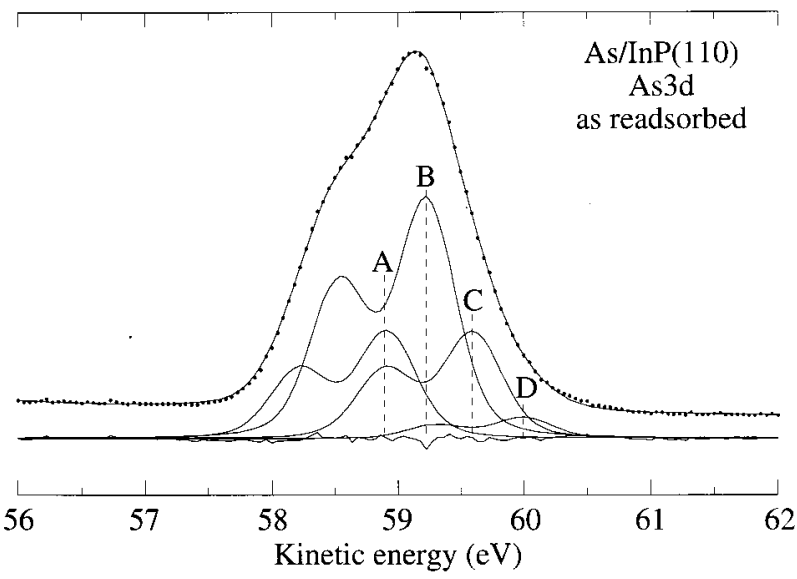

FIG. 3. As $3 d$ spectrum directly after redeposition. This is a fully optimized four-component fit of the same spectrum as in Fig. 1(h). Note the presence of component $D$ that is absent in Fig. 1(b) [and deliberately left out in the fit in Fig. 1(h)]. Fitting parameters as in Fig. 1.

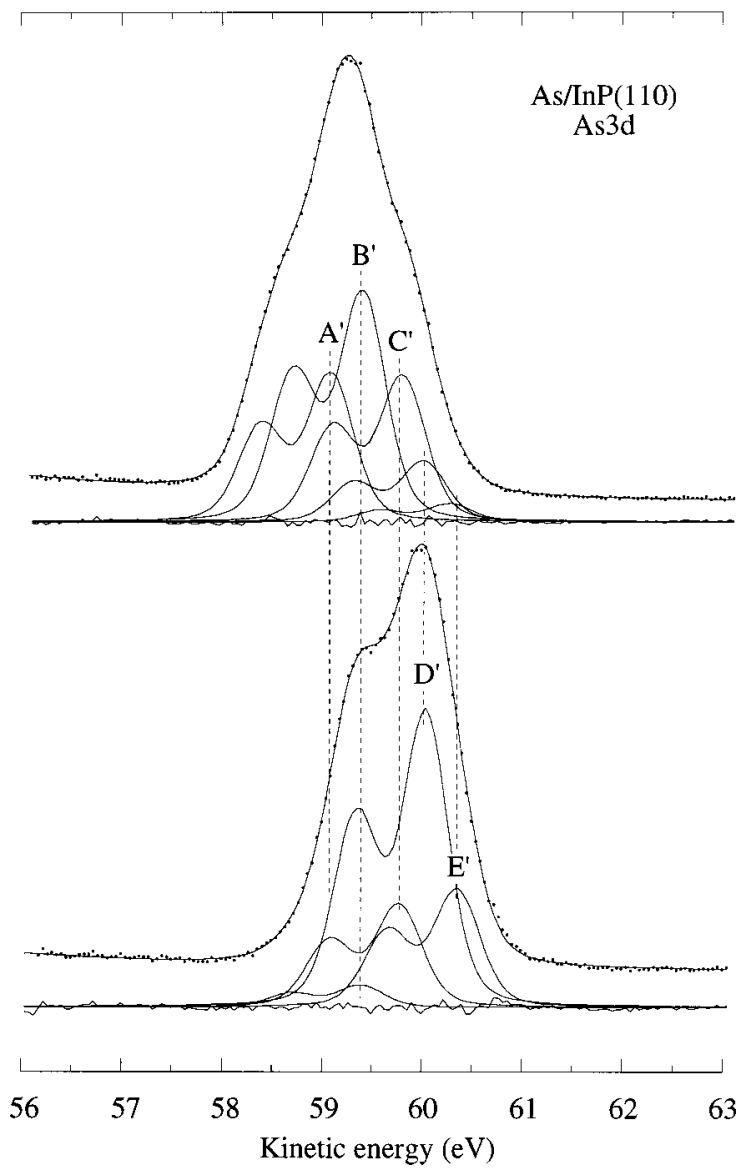

FIG. 4. As $3 d$ spectra from an "overheated" and restored InP:As crystal after As redeposition and subsequent annealing (see text).

distance away from the interface occupy electronically equivalent sites. Closer to the interface, a number of different adsorption sites are possible: atoms coordinated to surface anions or cations, atoms in a disordered layer on top of the first, polarized adlayer and atoms in higher adsorbed layers containing sites coordinated to other As atoms only. Although a definitive interpretation of the three components is not essential in the present context, one might suspect that the middle component $(B)$ is associated with atoms in the higher adsorbed layers and, considering the polarized nature of the substrate, the other two components with atoms coordinated to surface anions $(A)$ and cations $(C)$. In the subsequent analysis, however, we find that the relatively small attenuation of components $A$ and $C$ (as compared with that of substrate related emission, i.e., In $4 d$ ) suggest that they rather reflect sites within the As adlayer. Regardless of exactly what sites the components reflect, the three-component deconvolution works well to simulate adlayer desorption during the subsequent annealing process.

After a first annealing at about $280{ }^{\circ} \mathrm{C}$, the spectrum is apparently little affected. Detailed examination of the raw spectra reveals, however, a slightly more pronounced shoulder on the low-energy side and a tail developing toward the high-energy side. Decomposing this spectrum with the same fitting parameters as above, we find that components $A$ and $B$ are reduced in intensity relative to component $C$, and that new components on the high-kinetic-energy side have appeared. The new components $(D$ and $E$ ) obviously result 

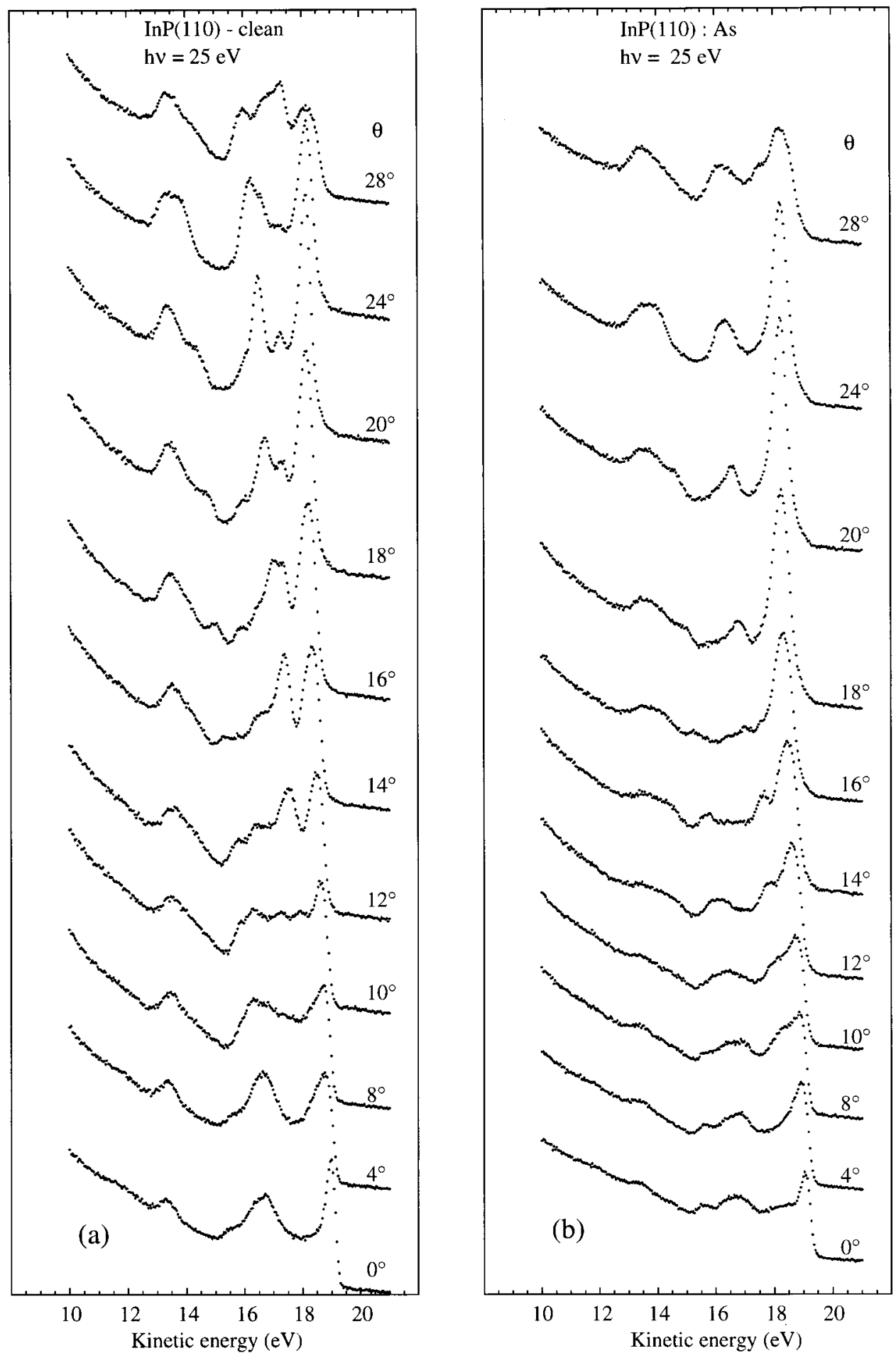

FIG. 5. Series of angle-resolved valence-band spectra excited with 25-eV photons from InP(110) in the "as cleaved" state (a) and after the P-As exchange reaction, corresponding to Fig. 1(g) (b). The two sets of data were recorded under identical experimental conditions.

from a thermally activated reaction. Table I summarizes the relative energies of the five components $A-E$.

With continued heating at elevated temperatures the spectrum undergoes a rather dramatic transformation. Using the same five components as above, we see that the transformation is a result of further reduction of the three initial components. By prolonged heating around $\sim 350{ }^{\circ} \mathrm{C}$, components $A-C$ are eventually almost fully removed, while components $D$ and $E$ remain relatively stable. It deserves pointing out that the spectrum obtained after the final annealing can be fitted almost equally well with one component. ${ }^{9}$ but only with substantially increased energy broadening. Limiting the broadening to the value dictated by the fitting of the In $4 d$ spectrum (which appears reasonable since the surface is very well ordered in this state), we must use at least two components. The two-component fit is supported by two further observations. The first one is a clear angular dependence, displayed in Fig. 2. We observe that the minimum between 


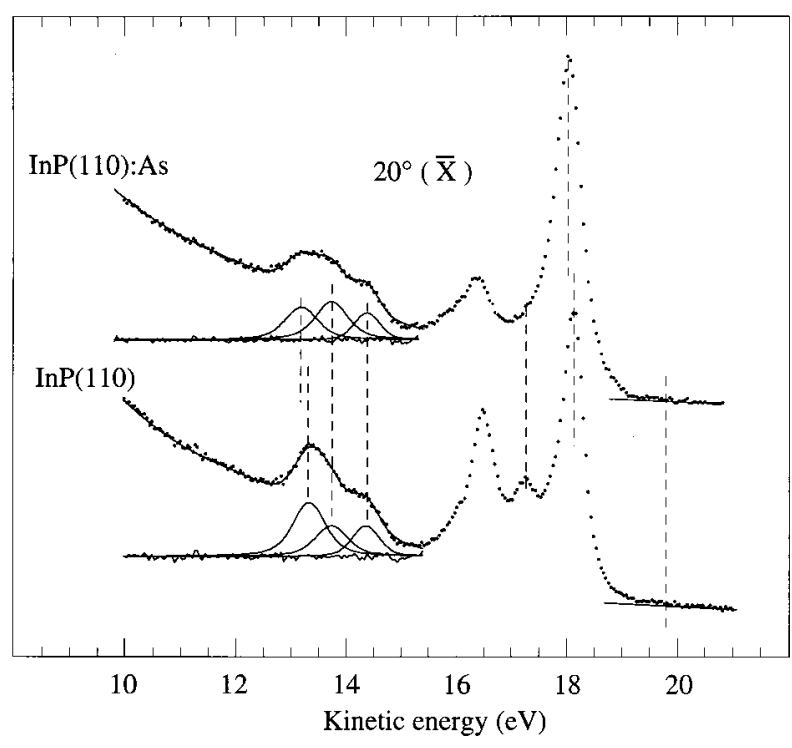

FIG. 6. Comparison of spectra recorded at $20^{\circ}$ emission angle, probing states around the $\bar{X}$ point in the surface Brillouin zone. The spectrum from the As-treated surface is shifted with $0.18 \mathrm{eV}$ toward lower kinetic energy, to account for As-induced band bending. The low-energy part of the spectra has been decomposed by means of a least-squares fitting routine.

the spin-orbit-split peaks becomes deeper at large emission angles, which we take as a sign of one component being reduced in relative intensity. Unfortunately, the energy separation between the two components is too small to reveal their presence more directly. Due to the small separation, the spectral decomposition becomes very delicate-the relative amplitudes depend sensitively on minute changes in the fitting parameters. Thus, from the fittings indicated in Fig. 2, we cannot make any conclusion regarding the geometry, even though the angular intensity dependence shown here would imply that the low-energy component derives from sites beneath those reflected by the high-energy component. (In a previous paper we actually presented an alternative fitting, including a very small additional component, which resulted in a quite different angular intensity dependence.) $)^{14}$ The second support for a two-component fit is found in the development of the spectrum after annealing above $500^{\circ} \mathrm{C}$, to be discussed below. This development also provides support for an assignment consistent with the angular dependence presented in Fig. 2; that is, component $E$ originates from atoms in the surface plane and component $D$ from atoms inside the crystal. It is worthwhile to note at this point that the In $4 d$ surface shift is somewhat reduced by the P-As exchange, from 0.30 to $0.26 \mathrm{eV}$. This change can be understood $^{15}$ as an effect of the somewhat lower electronegativity of As relative to $\mathrm{P}$, and the values agree well with those reported for In in InP and in InAs, respectively.

The above observations clearly show that the exchange

TABLE I. Energy separations (in eV) between the five As components used in the fits in Figs. 1-3.

\begin{tabular}{cccc}
\hline \hline$\Delta_{A-B}$ & $\Delta_{B-C}$ & $\Delta_{C-D}$ & $\Delta_{D-E}$ \\
\hline 0.33 & 0.36 & 0.36 & 0.25 \\
\hline \hline
\end{tabular}

reaction is activated only well above room temperature. This is in contradiction with the conclusions of Ref. 6, according to which the exchange is complete prior to any heat treatment. The experimental basis for this conclusion was observation of reversibility between poorly ordered and wellordered structures characterizing the as-adsorbed and annealed surfaces. In order to test this reversibility explicitly, the annealed and reacted surface was exposed a second time to $\mathrm{As}_{4}$. The LEED pattern from the re-exposed surface was very weak and with high background, indicative of a disordered overlayer. In this respect the situation is very similar to that after the first exposure. However, the As $3 d$ spectrum from the reexposed surface [Fig. 1(h)] shows that the process is not reversible in detail. Note that Fig. 1(h) does not show a fully optimized fit, but is included to illustrate that the three-component fit in Fig. 1(b) is not sufficient in the reabsorbed case. Even when allowed to relax somewhat from the exact peak positions and separations in Fig. 1(b), the threecomponent fitting is not satisfactory on the high-energy side (note that the fitting routine tends to compensate for the missing reacted components by raising the fitted curve systematically above the data points in the right part of the figure, which to some extent fills the gap created by the missing components). We see that the fit now requires additional components on the high-energy side, i.e., the annealing induced components $D$ and $E$. This emission is quite weak due to the As overlayer (components $A-C$ in Fig. 1). The degree of attenuation can be estimated by examination of the In $4 d$ emission, recorded at the same kinetic energy as the As $3 d$ signal, prior to and after As redeposition. We find that the attenuation of the annealing-induced components is approximately twice as strong as that of the In $4 d$ signal. With the interpretation of components $D$ and $E$ outlined above, one should expect that the surface component $E$ is shifted toward lower kinetic energy when the atoms in the surface plane interact with the As overlayer. It is reasonable to assume that the emission from As atoms coordinated to two in-plane In atoms and two As atoms should occur somewhere between the emission from As atoms coordinated to four In atoms (component $D$ ) and the emission from As atoms coordinated to four other As atoms [the single component in Fig. 1(a)], that is, somewhere in the region of components $B$ and $C$ in Fig. 1. This means that emission from the interface atoms would in this situation contribute to components $A-C$ (mainly to $B$ and $C$ ) and that only the unshifted component $D$ (reflecting As atoms in the bulk) would contribute to the emission from the part of the spectrum associated with the reacted components. In Fig. 3 we show a fourcomponent fit of the same raw data as in Fig. 1(h). Now the attenuated intensity of component $D$ is consistent with the corresponding attenuation of the In $4 d$ signal. Upon annealing of the reabsorbed surface the spectra undergo changes parallel to those observed during the first annealing cycle, and the two-component final spectrum is eventually restored. The final spectrum with two nearly equally strong components is quite stable up to $500{ }^{\circ} \mathrm{C}$. We note that the intensity ratio between the two components $D$ and $E$ appears to change systematically when the surface is depleted of As adatoms (as seen in Fig. 1). This agrees well with the preceding discussion attributing component $E$ to emission from sites only within the free surface. 
The difference between the spectra obtained after the first adsorption and after readsorption [Figs. 1(b) and 1(h)] demonstrates that the reaction-induced components are absent prior to annealing, and confirms our conclusion that the exchange reaction must be thermally activated. It is appropriate at this point to mention that in one case we did actually have to include a very small reaction-related component prior to annealing. We believe that this is due to active sites (defects) generated in the cleaving process. We have observed that the surface stoichiometry (as judged by the P/In core-level ratio) of different cleaves is not identical. Assuming that this is mainly due to $\mathrm{P}$ vacancies, it is plausible that the adsorbed As can to some extent be incorporated into the surface layer without any activation. In any case, the subsequent thermal treatment resulted in a significantly enhanced intensity of these components.

Annealing somewhat above $500{ }^{\circ} \mathrm{C}$ resulted in a surface with a very low As $3 d$ signal, while the In $4 d$ emission had a high-energy structure indicative of a metallic component. After annealing this surface in $\mathrm{As}_{4}$ atmosphere for about $1 \mathrm{~h}$ at about $330^{\circ} \mathrm{C}$, the metallic component was fully removed and the As signal restored. The P $2 p$ emission was at this point visible, but much weaker than prior to this treatment. This, in combination with the observation of a very good quality $1 \times 1$ LEED pattern and valence-band spectra with a well-developed angular dependence, leads us to conclude that a several-monolayers-thick InAs film is the result of this process. The surface was again exposed to $\mathrm{As}_{4}$, and during the following annealing As $3 d$ spectra were recorded (Fig. 4). These spectra were decomposed in qualitatively the same way as those discussed above, but allowing for small shifts due to the different substrate. The similarity between the different sets of data implies that the various components are of corresponding origins. In particular, we can then associate component $E$ with As atoms within the surface layer, and component $D$ with atoms in deeper layers. Thus the initial $\mathrm{P}$-As exchange process is not restricted to the first atomic layer.

We finally turn to the valence band data. Spectra from the clean $\operatorname{InP}(110)$ surface along the $\bar{\Gamma} \bar{X}$ azimuth are shown in Fig. 5(a). The spectra are in good agreement with those in a previous publication, ${ }^{11}$ which includes a detailed interpretation of the different features. In brief, all the prominent peaks were identified as surface states. The distinction between surface- and bulk-induced structures in angle-resolved photoemission can be based on several criteria. ${ }^{16}$ Nevertheless, there are numerous examples where the interpretation of particular features can be questioned. In the case of clean $\mathrm{InP}(110)$, some of the weaker structures could not be assigned unambiguously. The possibility of selective exchange of one of the surface species without modifying the surface geometry opens a new, complementary method in this context. In contrast to the "normal" surface contamination test, this kind of surface perturbation should leave the bulkderived emission unaffected, since surface diffraction effects should be the same prior to and after the exchange.

In Fig. 5(b) we show a set of valence-band spectra from the same surface as in Fig. 5(a), but after the P-As exchange [corresponding to the core-level data in Fig. 1(g)]. As pointed out earlier, the two sets of spectra show strong similarities. There are, however, some significant differences. We see, for instance, that the small peak around $17 \mathrm{eV}$ in the $20^{\circ}$ spectrum in Fig. 5(a) appears to be missing in Fig. 5(b). The differences between the two spectra are, however, more subtle. In order to clarify the situation, the spectra must be compared in detail, on an energy scale that compensates for band bending changes. In Fig. 6 we present such a comparison for the pair of spectra obtained at a $20^{\circ}$ emission angle (probing states around the $\bar{X}$ point in the surface Brillouin zone). We find that most of the spectral features are shifted by around $0.10-0.15 \mathrm{eV}$ toward higher binding energy after the P-As exchange. (It should be mentioned that this shift is of about the same magnitude, but of opposite sign than that reported in Ref. 9. The reason for this discrepancy is unclear at this point.) Having aligned the spectra with respect to the bulk core levels, all bulk-derived valence-band features should appear unshifted. There are few such structures directly visible in the two spectra in Fig. 6. The broad peak around $6 \mathrm{eV}$ below the valence-band maximum (VBM) should contain a bulk component reflecting the high density of states at the $X_{3}$ point of the bulk Brillouin zone. From the data, however, it is clear that the observed structure contains several components, one of which is a surface state. ${ }^{11}$ By applying the least-squares fitting routine used in the analysis of the core-level data to the $20^{\circ}$ spectrum, we find that the broad peak can be separated into three components, one of which reflects the rapidly downwards dispersing peak seen in Fig. 5.

The comparison between the two spectra now shows that the upper two components of the 6-eV structure coincide in energy, but the lowest one shifts downwards upon As exchange. Accordingly, we conclude that the lowest of the three peaks represents a surface state. Unfortunately, while shifted peaks directly indicate surface states, the argument cannot be turned around to say that unshifted ones are bulk derived. The nondispersive behavior of the middle peak, however, suggests that it reflects a high density of states in the bulk at $X_{3}$. According to previous investigations, ${ }^{17}$ the $X_{3}$ point in InP is located at $6.0 \mathrm{eV}$ below the VBM. We have marked the expected VBM position with a dashed line at $19.7-\mathrm{eV}$ kinetic energy. Indeed, a detailed examination reveals a weak, steplike intensity change in this region, which most likely reflects the onset of emission at the VBM. This observation provides support for the above assignment of the density-of-states peak, and accordingly for the identification of the lowest peak.

The rapidly dispersing peak, found at $14.4-\mathrm{eV}$ kinetic energy in the $20^{\circ}$ spectra, was previously assigned as a surface state. Since the peak is unshifted, this assignment is not confirmed by the present analysis, but nor is it contradicted.

In the high-kinetic-energy part of Fig. 6, we see that the two dominant peaks are also shifted toward lower kinetic energies in the As-exchanged case. The surface origin of these peaks was established earlier. The small peak that shows up distinctly between the two strong peaks in the $20^{\circ}$ spectrum from untreated InP, appears at first sight to be absent after P-As exchange. With the spectra correctly aligned, we interpret the absence of this peak as an effect of the overall downwards shift of surface-related peaks, in combination with broadening of the uppermost surface state peak. In fact, careful examination of the low-energy side of the leading peak suggests the existence of a small shoulder, 
which we believe corresponds to the weak peak. Thus there is reason to suspect that the small peak is bulk related. One obvious candidate explanation in this case would be the high density of states at the $X_{5}$ point. Since the $X$ point of the bulk Brillouin zone is projected at $\bar{X}$, this would account for the relatively distinct appearance of this structure in the $20^{\circ}$ spectrum. Using the $X_{3}$ peak as reference, we would then obtain for the $X_{5}$ point an energy of $2.5 \mathrm{eV}$, in disagreement with literature data ${ }^{16}(2.2 \mathrm{eV})$. Considering the large discrepancy, we believe that the peak should be assigned as a surface state, just as proposed in the previous work. ${ }^{11}$

\section{SUMMARY}

In summary, our core-level data from As deposited on $\operatorname{InP}(110)$ show that the adsorbate-substrate interaction is not strong enough to promote a P-As exchange reaction at room temperature. An exchange is observed, but only at elevated temperatures. The process appears to be completed below the temperature of As adlayer desorption. Our conclusions are

*Present address: Department of Physics, Hong Kong University of Science \& Technology, Clear Water Bay, Kowloon, Hong Kong, China.

${ }^{1}$ P. Skeath, C. Y. Su, W. A. Harrison, I. Lindau, and W. E. Spicer, Phys. Rev. B 27, 6246 (1983).

${ }^{2}$ R. M. Feenstra and P. Mårtensson, Phys. Rev. Lett. 61, 447 (1988).

${ }^{3}$ B.-J. Schäfer, A. Förster, M. Londschien, A. Tulke, K. Werner, M. Kamp, H. Heinecke, M. Weyers, H. Lüth, and P. Balk, Surf. Sci. 204, 485 (1988).

${ }^{4}$ J. P. LaFemina, C. B. Duke, and C. Mailhiot, J. Vac. Sci. Technol. B 8, 888 (1990).

${ }^{5}$ Z. Q. He, Y. O. Khazmi, J. Kanski, L. Ilver, P. O. Nilsson, and U. O. Karlsson, Phys. Rev. B 52, 16602 (1995).

${ }^{6}$ P. V. Santos, B. Koopmans, N. Esser, W. G. Schmidt, and F. Bechstedt, Phys. Rev. Lett. 77, 759 (1996).

${ }^{7}$ S. Mankefors, P. O. Nilsson, J. Kanski, and K. Karlsson, Phys. Rev. B 56, 15847 (1997).

${ }^{8}$ A. Tulke and H. Lüth, Surf. Sci. 211/212, 1001 (1989). supported by two experimental results: (a) direct observation of the appearance of core-level photoemission from the exchanged As; and (b) observation of different spectra after the first As adsorption and after annealing followed by readsorption, the latter clearly showing that the surface conditions are irreversibly modified by the annealing. The results also show that the P-As exchange is not limited to the outermost layer only.

We have also studied photoelectron spectra from the valence band, and found large similarities between the clean InP surface and that after P-As exchange. The differences, albeit small, can be used for the identification of surface states.

\section{ACKNOWLEDGMENTS}

The project was supported by the Swedish Natural Science Research Council. One of the authors (Z. Q. He) is indebted to the Swedish Institute for financial support.

${ }^{9}$ T. Chassé, G. Neuhold, and K. Horn, Surf. Sci. 331-333, 511 (1995).

${ }^{10}$ U. O. Karlsson, J. N. Andersen, K. Hansen, and R. Nyholm, Nucl. Instrum. Methods Phys. Res. A 282, 553 (1989).

${ }^{11}$ H. Qu, J. Kanski, P. O. Nilsson, and U. O. Karlsson, Surf. Sci. 255, 237 (1991).

${ }^{12}$ W. G. Wilke, V. Hinkel, W. Theis, and K. Horn, Phys. Rev. B 40, 9824 (1989).

${ }^{13}$ N. Esser, D. R. T. Zahn, C. Müller, W. Richter, C. Stephens, R. Whittle, I. T. McGovern, S. Kulkarni, and W. Braun, Appl. Surf. Sci. 56-58, 169 (1992).

${ }^{14}$ L. Ilver, J. Kanski, P. O. Nilsson, H. Oscarsson, M. Davilà, and U. O. Karlsson, Appl. Surf. Sci. 123/124, 95 (1998).

${ }^{15}$ A. B. MacLean, J. Phys.: Condens. Matter 2, 1027 (1990).

${ }^{16}$ G. V. Hansson and R. I. G. Uhrberg, Surf. Sci. Rep. 9, 197 (1988).

${ }^{17}$ G. P. Williams, F. Cerrina, G. J. Lapeyre, J. R. Anderson, R. J. Smith, and J. Hermanson, Phys. Rev. B 34, 5548 (1986). 